\section{Lift the Lip: A Simple Visual Tool for Caries Risk Assessment}

Early childhood caries (ECC) is the tooth decay present in the primary teeth of children under 6 years of age. ECC may begin soon after primary teeth erupt and white spot lesions are its earliest manifestation that can progress into cavitated carious lesions within a very short time. ECC management is demanding, requiring substantial cooperation from young children. Often due to the severity of the condition, comprehensive care under general anesthesia is required, which is expensive and carries an inherent risk [1].

Early identification and management of ECC can reverse the disease process and prevent further decay. The first oral examination is therefore recommended soon after the eruption of first tooth and no later than the age of one year. This early visit helps in evaluating the caries risk of the child, and imparting oral health education as well as anticipatory guidance to parents [2]. Most parents are not conscious of the benefits of this early oral examination and often see a dentist only when severe ECC has already set in. Infants and toddlers are likely to be seen by pediatricians more frequently. If these health professionals can spot children with a higher risk for caries and refer them to a dentist, early preventive measures can be initiated.

'Lift the lip' maneuver has been developed as a simple, visual screening tool for caries risk assessment [3]. It is particularly useful in young children because till 18 months of age, there is greater prevalence of white spot, non-cavitated lesions that can be arrested or reversed by preventive interventions. Beyond this age, cavitated lesions requiring surgical mediation become more common [4]. Timely detection and referral of high-risk children by pediatricians may be the key to control ECC through preventive, non- invasive measures that are pain-free.

\author{
Namrata C Gill \\ Department of Pedodontics and Preventive Dentistry \\ Dr Harvansh Singh Judge Institute of Dental Sciences \\ Panjab University, Chandigarh. \\ namratagill@gmail.com
}

\section{REFERENCES}

1. Policy on early childhood caries (ECC): Classifications, consequences, and preventive strategies. Pediatric Dent. 2016;38:52-54.

2. Arrow P, Raheb J, Miller M. Brief oral health promotion intervention among parents of young children to reduce early childhood dental decay. BMC Public Health.2013;13:245.

3. Nicolae A, Levin L, Wong PD, et al. Identification of early childhood caries in primary care settings. Paediatr Child Health. 2018;23:111-15.

4. Bach K, Manton DJ. Early childhood caries: A New Zealand perspective. J Prim Health Care. 2014;6:169-74.

\section{Leprosy Mimicking Carpal Tunnel Syndrome in a Child on Growth Hormone Therapy}

A 16-year-old boy, operated for craniopharyngioma at 12 year was receiving thyroxine, hydrocortisone and desmopressin for last 4 years, and growth hormone $(\mathrm{GH})$ at $(0.25 \mathrm{mg} / \mathrm{kg} /$ week $)$ for 1 year, He presented with increasing pain, numbness and tingling over right palm for 3 months. On examination, swelling involving right wrist and hand, and claw hand deformity were noted. Right hand was cooler than the left, and radial artery pulsations were feeble. As the child was on GH, with carpal tunnel syndrome (CTS) being a known adverse effect, this was the initial diagnosis. GH therapy was withheld and physiotherapy was initiated.

On the next visit, detailed clinical examination showed decreased sensory perception, a small hypopigmented macule over dorsal aspect of ring finger, and thickened ulnar nerve behind right medial epicondyle, raising the possibility of leprosy. Further review of history revealed that mother had been treated for leprosy 20 year back. Nerve conduction study showed right median and ulnar motor sensory neuropathy, and skin biopsy revealed perivascular lymphocyte infiltration with few epithelioid cells, confirming a diagnosis of tuberculoid leprosy. Multidrug therapy (MDT) for leprosy was initiated and GH was restarted. At follow-up after 12 months of MDT, there was complete resolution of symptoms.

In children, the commonest cause of CTS is lysosomal storage disorder [1]. It is reported in $35-45 \%$ of acromegalic patients and $5-30 \%$ of adults receiving GH therapy; however, it is rare in children [2]. The mechanism is related to salt and water retention by growth hormone, leading to edema of synovial tissue and compression of median nerve [2]. In leprosy, the neuropathy is due to chronic inflammatory granuloma [3]. In settings where leprosy is rare, the diagnosis may be missed. Koss, et al. [4] previously reported a young woman, who presented with recurrent CTS despite surgical decompression and was later diagnosed to have leprosy [4]. In our case also, the child being on GH therapy initially mislead us towards considering CTS. In clinical medicine, sometimes the most likely possibility may not be the correct answer. 